\title{
Extended Foucault Method for External Magnetic Fields with Conventional TEM
}

\author{
Hiroshi Nakajima ${ }^{1}$, Atsuhiro Kotani ${ }^{1}$, Ken Harada ${ }^{1,2}$, Yui Ishii ${ }^{1}$ and Shigeo Mori ${ }^{1}$ \\ 1. Department of Materials Science, Osaka Prefecture University, Sakai, Osaka 599-8531, Japan \\ 2. Center for Emergent Matter Science, RIKEN, Akanuma, Hatoyama, Saitama 350-0395, Japan
}

Foucault method as well as Fresnel method has been used to observe magnetic domains in Lorentz microscopy [1]. In Foucault method, magnetic domains are visualized by selecting spots due to the magnetization. It had been considered that Foucault method required a specially dedicated TEM whose lens was a magnetic-field shielding type (Lorentz TEM). However, an electron optical system for Foucault imaging was constructed recently using a conventional TEM without any special equipment by making a crossover on the selected area (SA) aperture plane [2]. In addition, the electron optical system enabled you to perform small angle electron diffraction (SmAED) mode, switching from Foucault imaging mode. Although Foucault method was realized with a conventional TEM, the current of the condenser lens could not be changed to fix the crossover position on the SA aperture plane.

Here we report an extended optical system with an objective mini-lens in order to control the illumination and imaging systems independently. The total amount of the electron beam on the observed specimen can be adjusted adequately. Furthermore, this electron optical system make it possible to perform Foucault method and SmAED in external magnetic fields without changing the illumination system. We succeeded in observing magnetic domain structures and their structural change by applying an external magnetic field in the rhombohedral phase of $\mathrm{La}_{0.7} \mathrm{Sr}_{0.3} \mathrm{MnO}_{3}$ (LSMO) using the present electron optical system.

Figure 1 shows schematic illustrations of the constructed electron optical system of (a) SmAED and (b) Foucault imaging modes. The objective mini-lens and condenser lens are tuned in order to converge electron beam on the SA aperture plane. The aperture works for selecting the deflected electron beams. Since the objective lens (OL) is switched off in normal Lorentz microscopy observation, external magnetic fields can be applied to the observed specimen by exciting the OL. When the OL is excited, the current of the objective mini-lens is adjusted so as not to move the position of the crossover. The SmAED mode can be switched from the Foucault imaging mode by decreasing the current of the intermediate lens 1 (Fig1. (b)). The camera length and image magnification are adjustable by changing the focal length of the image forming lens under the SA aperture.

The SmAED pattern of LSMO with a camera length of $150 \mathrm{~m}$ is shown in Fig. 2(a). The 000 direct spot was split by the magnetic deflection due to the $180^{\circ}$ magnetic domains with magnetic moments antiparallel between two neighboring domains. The magnetic field due to the magnetization can be calculated from the split angle and the value is $0.5 \mathrm{~T}$, which is consistent with the magnetic moment of Manganese. The Foucault images obtained selecting one of two split spots are shown in Figs. 2(b) and 2(c). The magnetic field of $50 \mathrm{mT}$ was applied parallel to the optical axis in Fig. 3. An extra spot appeared in the SmAED pattern, in addition to the two split spots in Fig. 3(a). Magnetic domains generated by the external magnetic fields were visualized in Foucault images of Figs. 3(b) and 3(c). The deviation angle of the emerged magnetization (yellow arrows) from the original direction was derived to be $22^{\circ}$ by measuring the azimuthal angle in Fig. 3(a). As demonstrated here, the quantitative analysis of the magnitude and the direction of the magnetization is one of some advantages of SmAED method. 
References:

[1] C N Chapman, J. Phys. D; Appl. Phys. 17 (1984), 623.

[2] Y Taniguchi et al., Appl. Phys. Lett. 101 (2012), 093101.

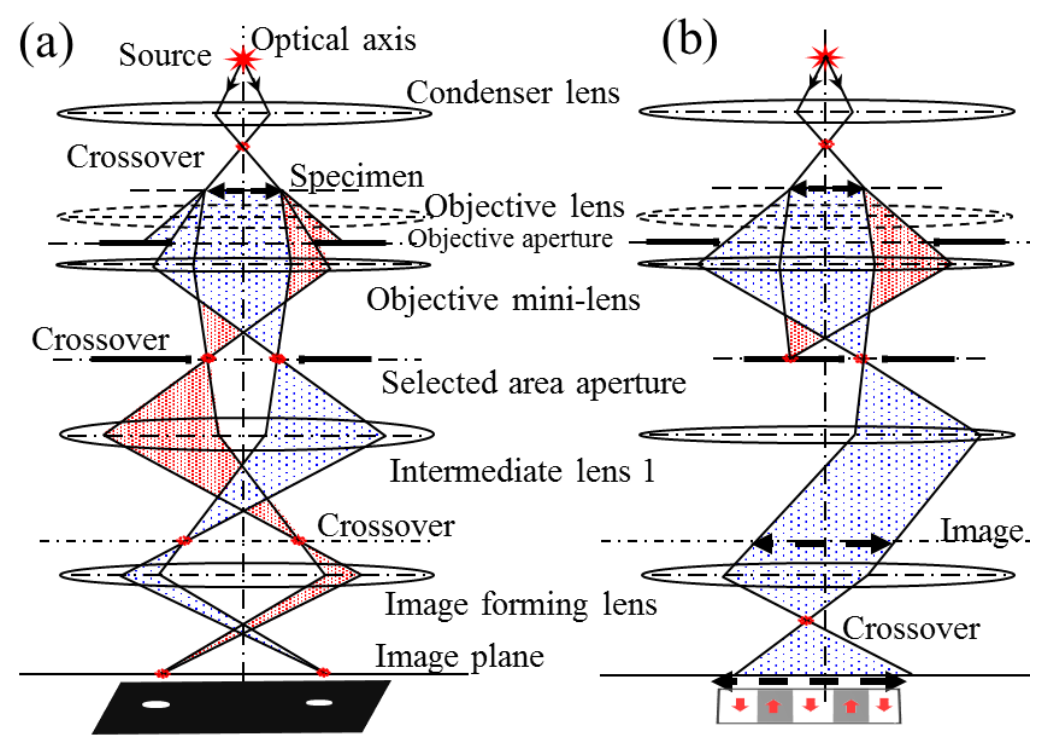

Figure 1. Schematic illustrations of electron optical systems of (a) small angle electron diffraction mode and (b) Foucault mode.

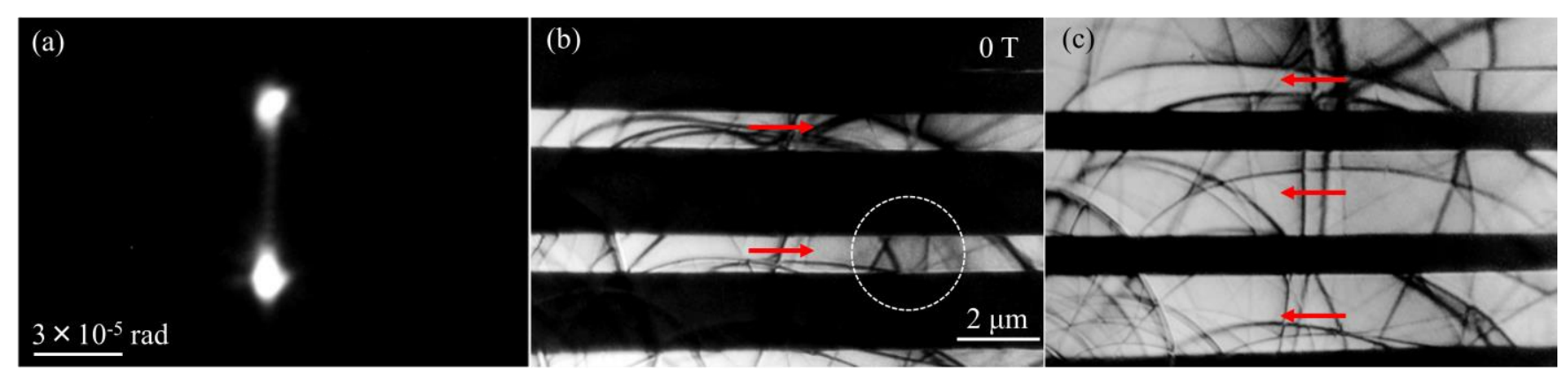

Figure 2. (a) SmAED pattern in LSMO under no magnetic field. Foucault images obtained using (b) the upper and (c) lower spot in (a). The arrows show the direction of the magnetization in each magnetic domain. The SmAED pattern (a) was obtained from the region indicated by the dotted circle.

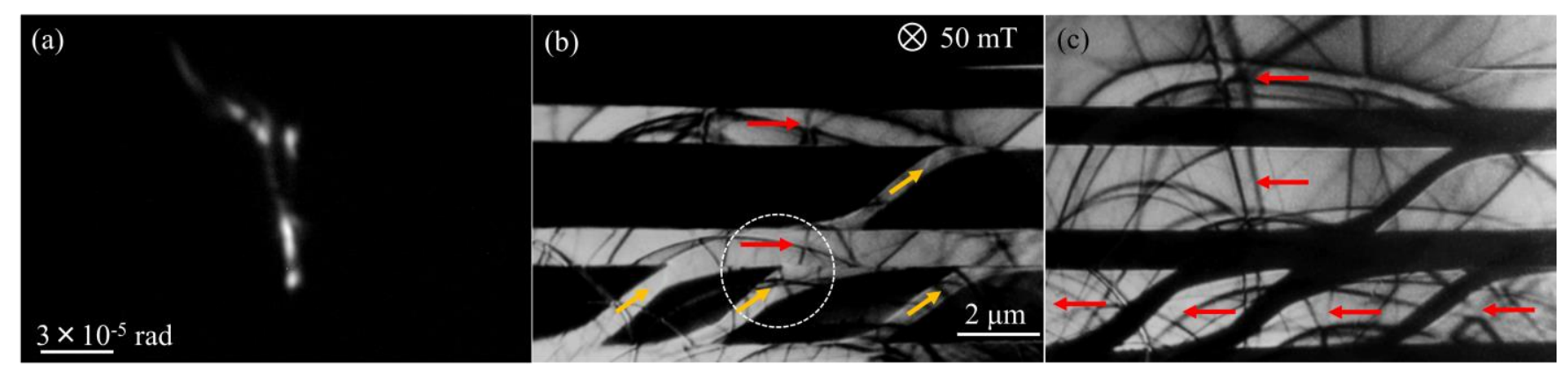

Figure 3. (a) SmAED pattern in LSMO at $50 \mathrm{mT}$. Foucault images obtained using (b) the upper two spots and (c) lower spot in (a). Yellow arrows show the direction of the magnetization generated by applying the external magnetic field. 